\title{
LUNG RESECTION FOR PULMONARY TUBERCULOSIS
}

BY

\author{
B. J. BICKFORD, F. RONALD EDWARDS, J. R. ESPLEN, J. HAMILTON \\ GIFFORD, A. M. MAIR, AND O. F. THOMAS
}

From the Aintree and Broadgreen Centres of the Liverpool Regional Thoracic Surgical Service and the Department of Surgery, University of Liverpool

(RECEIVED FOR PUBLICATION OCTOBER 31, 1950)

In spite of the time and endeavour spent upon the treatment of pulmonary tuberculosis, it is far from the truth to say that the results of treatment are generally satisfactory.

This statement is underlined by the figures of Thompson $(1942,1943)$, who found that $42 \%$ of a group of 406 patients with a positive sputum in the County of Durham were dead within 12 months of diagnosis, while only one patient in eight survived for 10 years. Cox (1936) and Bradbury (1946) give figures from the Lancashire County Council which showed somewhat similar results. Of 1,230 cases of pulmonary tuberculosis notified in $1930,63.1 \%$ had died of their disease within five years, and of the 1,178 cases notified in $1940,58.3 \%$ died in a similar period. When it is considered that $27.9 \%$ of the cases notified in 1930 and $33.6 \%$ of the cases notified in 1940 were still under treatment at the end of five years, the figures are discouraging indeed.

Stocks and Lewis-Faning (1944) conclude that, in spite of a rapid fall in the number of notifications of disease in the period from 1923 to 1939, the average expectation of a patient eventually dying of his disease remained unchanged.

It would not be fair to conclude from these figures that the prognosis of certain types of cases has not improved, and, in particular, the study of patients treated by thoracoplasty shows reason for optimism. Hurford (1941) published details concerning a group of 67 patients treated by thoracoplasty and a similar number who declined this treatment when it was offered. On follow-up for a minimum period of one year, $65 \%$ of operated cases were quiescent compared with $16 \%$ of the controls ; only $18 \%$ of operated patients, but $45.5 \%$ of the controls, were dead. Sellors (1947) recorded sputum conversion in $84 \%$ of 633 patients treated by thoracoplasty between 1935 and $1946 ; 59.2 \%$ were fit for full work and $12.1 \%$ were dead. Similar figures are given by other authors.

Over a period of years certain views have been developed on the pathology and treatment of this disease which will be discussed, and as a development from these a more direct attack on the diseased area in the lung has been adopted which it is hoped may produce some improvement on these figures. 


\section{Chemotherapy and Collapse Therapy}

Treatment by streptomycin and P.A.S. is beneficial in certain types of acute disease, but chronic tuberculous infection of the lungs is not influenced by it. It is even doubtful whether the ultimate outlook of the favourably affected cases is any better, but it is probable that it is only a matter of time before a truly specific agent against the tubercle bacillus in man is developed. Even though such an agent may sterilize the affected areas, surgical treatment will still be required to extirpate lung tissue so severely damaged as to be liable to recurrent secondary infection.

The sheet anchor of definitive treatment of pulmonary tuberculosis has been collapse therapy. We have rather slowly come to realize that collapse therapy only gives satisfactory results when it is manifestly successful in controlling the lesion, self-evident though this statement may seem at first sight to be. The course of the disease is so variable and unpredictable that most clinicians will be able to recall cases in which a far from perfect artificial pneumothorax has produced clinical improvement and perhaps quiescence maintained over a long period. In general, however, the only satisfactory artificial pneumothorax is the one in which good concentric relaxation of the lung results in closure of cavities, and when there is a high degree of natural resistance on the part of the patient.

The aim of collapse therapy is to produce such relaxation of the lung as will only allow slight movement during respiration, and will allow tuberculous cavities to close. The conditions thus produced favour healing, and the body's defences are strengthened by the general circumstances of sanatorium life. Nevertheless, it is evident that the healing of a cavitating tuberculous lesion is an uncertain process under any circumstances, and lesions apparently well healed and calcified may break into renewed activity many years later under conditions of stress.

It is usual to assume that the conversion of sputum from positive to negative is a criterion of healing, but the change may take place so rapidly after a successful artificial pneumothorax or thoracoplasty that this cannot really be the case. It is more likely to be due to bronchial occlusion. Such occlusion in an uncollapsed case may also give a negative sputum that belies the concealed activity of the disease. A caseous nodule, or " tuberculoma" as some prefer to call it, may empty intermittently with the production of a positive sputum, and innumerable tubercle bacilli may be seen within such a lesion after it has been resected.

The negativity of the tuberculous patient's sputum is to some extent relative, so that many cases negative on direct smear examination prove to be positive after concentration, culture or guinea-pig inoculation of sputum, or of bronchoscopic or laryngeal swab material. Conversion of the sputum from positive to negative nevertheless remains the only useful prognostic guide as to the success of treatment, and Livingstone (1949) has shown that this is true of a large number of cases treated by all forms of collapse therapy and followed for 10 years after diagnosis. Of those without sputum conversion $94.5 \%$ had died, but $67.2 \%$ with sputum conversion were still living.

\section{Natural History of the Disease}

In spite of the good results obtained by collapse therapy in suitable cases, only a small proportion of patients admitted to a sanatorium ever become suitable for 
major surgery. All too often, long years are spent in a struggle to obtain control over bilateral lesions, only for defeat to be acknowledged in the end.

Through improved methods of early diagnosis it is to be hoped that fewer cases of disease will be first recognized in the bilateral state.

It is almost certain that the adult form of pulmonary tuberculosis is essentially an inhalational disease which is usually confined in the first place to a strictly limited portion of the lung, commonly one upper lobe. This statement is perhaps an over-simplification of a rather complex process, but it is, we believe, in the main true. This is not to say that infection may not be at some time fairly widespread, but usually the tissue defences are able to deal with the greater part of the disease. Where cavitation occurs, a process indicating in the lung a failure of the natural process of healing, it is commonly at first in one or two quite localized areas. Once cavitation has taken place the chances of spontaneous healing are diminished, and will in any event be slow. From areas of established uncontrolled cavitation spread to other parts of the lung is almost certain to take place sooner or later.

We do not believe that tuberculosis is necessarily and at all stages a generalized disease in the same sense that syphilis is. It is the region or organ in which the natural defences have failed to overcome infection that is the object of attack by surgical methods in tuberculosis of the lung or elsewhere.

\section{RESECTION OF THE LUNG}

When reviewing the history of cases of chronic and advanced pulmonary tuberculosis, it is striking in how many of them the disease has, at one time, been limited to a single lobe. The question naturally comes to mind, If the diseased area could have been excised at that time, would not progression to a hopeless condition have been avoided?

The first successful extirpation of the lung for pulmonary tuberculosis was carried out by Sir William Macewen in 1906, but it is only in the last few years that pulmonary lobectomy and pneumonectomy have become safe surgical procedures for the patient.

The idea of being able to remove all grossly diseased lung tissue instead of performing a thoracoplasty makes a natural appeal both to the surgeon and to the patient, but early attempts in this direction were not encouraging. Dolley and Jones (1940), Churchill and Klopstock (1943), Sweet (1946, 1950), Overholt, Langer, Szypulski, and Wilson (1946), Overholt, Wilson, Szypulski, and Langer (1947), and Gale, Dickie, and Curreri (1949) have all reported considerable numbers of cases in the U.S.A., and Sellors and Hickey (1949) have reported on their own experiences in this country.

The early results were marred by the occurrence of serious complications, the chief of which were bronchopleural fistula, the spread of infection to other parts of the lungs, to the pleura or chest wall, and reactivation of pre-existing disease. Later improvements in surgical and anaesthetic technique and in the general use of streptomycin during the post-operative period have diminished the incidence of such disturbing complications. Overholt and his colleagues (1946) reported two fatalities in 35 lobectomies and nine in 69 pneumonectomies; Gale and others (1949) had two deaths in 33 lobectomies and 47 pneumonectomies; and Sellors 
and Hickey (1949) had five fatalities in 55 lung resections undertaken for known tuberculosis.

Long-term results are not yet available. Sweet (1950) has reported that, of a series of patients treated by lung resection and followed up for at least three years, 19 out of 54 patients who survived operation remained well. This total comprised six $(19 \%)$ of 25 lobectomies, and $13(45 \%)$ of 29 pneumonectomies ; about twothirds of all the cases showed activity of other tuberculous foci after the operation. Nevertheless, it seems to us that such figures are unduly pessimistic, and do not reflect what may be expected from present-day procedures when streptomycin is freely available and advances have been made in the technique of operation and in the selection of cases for surgery.

\section{The Present Investigation}

Because of their failure to respond to collapse therapy, certain types of tuberculous infection have been generally accepted as suitable for resection. These include a patent cavity in a lung well collapsed by a pneumothorax, failed thoracoplasty, tuberculous bronchiectasis, the caseous nodule or "tuberculoma," giant cavities, basal cavities, "destroyed lung," and bronchostenosis. The work of Sarot (1949) has demonstrated that in tuberculous empyema resection of the pleura with the underlying diseased lung tissue is a satisfactory procedure. These conditions make up only a small proportion of the cases that might be treated surgically, and in our work at Aintree and Broadgreen Hospitals in Liverpool we have sought to discover to what extent resection could be made more generally applicable to the treatment of pulmonary tuberculosis.

Bearing in mind the considerations we have discussed, it appears to us to be an unassailable argument that when a tuberculous lesion has progressed to such an extent that the chances of ultimate healing are uncertain under any other form of treatment, and it yet remains localized to a part of the lung technically removable by surgery, the ideal treatment is resection. For only in this manner can hopelessly diseased tissue be removed and further spread of infection be avoided, and the patient be given a chance to overcome any lesser areas of infection by his natural defences. Only thus can the long period of treatment necessary after the establishment of an artificial pneumothorax be avoided, and the anxiety that is so often experienced when an artificial pneumothorax is abandoned because of complication, or because it is thought to have been maintained for long enough, banished.

We reasoned that, if the mortality and morbidity rates of lung resection could be shown to be low, the optimum time for operation would appear to be at an early stage in the disease whenever its extent and nature make the eventual outcome a matter for serious doubt. A period of observation and preliminary treatment remains necessary in every case, during which areas of exudative disease could be expected to show improvement and perhaps to heal. If successful, treatment by resection might be expected to show a reduction in the period required to heal the individual case.

We have accordingly treated by resection cases which in the past we would have submitted to thoracoplasty. These include all forms of fibro-caseous and cavernous disease which in the opinion of our clinical conferences were unlikely to be finally healed by any simpler form of treatment. 
Because of these differences from previous policy, it will not be accurate to compare results achieved during the present investigation with those obtained by thoracoplasty. When larger numbers have been treated, it will be possible to analyse the figures in detail, but it is not helpful to do so at the present stage.

The implementation of this policy does not mean that lesser measures of collapse therapy have been abandoned. Artificial pneumothorax is reserved as far as possible for those cases in which it appears likely to be in all respects satisfactory, and is only exceptionally induced or maintained in other circumstances.

\section{Selection of Cases and Pre-operative Treatment}

Because of the danger of reactivation of incompletely healed foci of disease left behind after operation and subjected to the strain of possible hyper-expansion, it is clear that care must be taken to evaluate the degree of activity of lesions in all parts of the lungs before operation.

The presence of bilateral disease has always made it necessary to exercise caution when selecting patients for major surgical treatment, and greater care is necessary when resection is to be employed rather than thoracoplasty. We have adopted a working generalization that resection should only be undertaken in bilateral disease when the lesion on the better side has been observed to remain stable over a period of at least 12 months.

We have ourselves confined the use of pneumonectomy to predominantly unilateral disease, but two of the lobectomies reported here were performed in the presence of a contralateral artificial pneumothorax.

More recently, the immediate successes of segmental resections have enabled us to contemplate and undertake the extirpative treatment of bilateral disease where the areas involved on each side were comparatively small.

At weekly conferences attended by all members of the staff and by tuberculosis physicians of the region, a general plan of treatment is laid down at an early stage for patients who appear likely to need major surgery. Because of the length of the waiting-list for operation, temporary collapse measures may be necessary, but performance of these should not prejudice the definitive operation. The advisability of temporary collapse measures needs careful consideration in order not to interfere with the success of a projected major operation in any way. In the presence of bilateral disease an artificial pneumothorax may be induced over a lesion in the better lung if it does not seem to be sufficiently stable to tolerate an operation on the opposite side. It is often difficult to decide what to do in such a case when a pneumonectomy is projected. On the one hand, a pneumothorax which is not completely satisfactory may result in the development of an effusion or of an adhesive pleuritis which may severely limit respiratory function, and, on the other, it may be difficult to maintain a small pneumothorax during the post-operative period. For these reasons it may be wise to proceed with the major operation and to induce the pneumothorax afterwards rather than to induce a "shallow" pneumothorax beforehand.

When a resection is planned for a lesion uncontrolled by a well-established artificial pneumothorax we do not usually advise abandoning the pneumothorax before operation as long as there is no ill effect resulting from it. 
A temporary pneumoperitoneum, with or without chemotherapy, is frequently helpful in toxic cases and in those with actively progressing lesions. It may be supplemented by a phrenic nerve crush if necessary, but this should not as a rule be performed on the better side when a lung resection is a future possibility.

Apart from the vital capacity, which is recorded in every case, tests of pulmonary function are not performed as a routine. Bronchospirometry has proved useful in certain cases, particularly when it is desired to study the function of the individual lungs in the presence of emphysema or adhesive pleurisy.

When there is a chronic empyema, hypoproteinaemia is corrected, for patients in this condition will not stand a long operation. A mild degree of amyloidosis is not a contraindication to operation.

Tomography of the lungs should be undertaken before operation whenever possible and particularly when there is doubt about the exact extent of disease. Tomographs of poor quality may be misleading and are of less value than the standard postero-anterior and lateral films. A lordotic view has been found to be very informative in order to throw the opposite apex into relief.

Before a patient is finally accepted as suitable for operation bronchoscopy is carried out. Active endobronchitis at the proposed site of division of a bronchus is a contraindication to operation. In such a case, a further bronchoscopy is performed after a course of treatment by streptomycin and P.A.S., and operation is reconsidered if the local condition appears to have healed.

As explained below, we use a pneumoperitoneum to help in preventing mediastinal displacement, particularly after a pneumonectomy; therefore a previously induced therapeutic pneumoperitoneum is continued. Otherwise, it is our practice to induce one at least a week before operation, and if possible earlier than this in order that it should become well established.

It is generally accepted that the use of streptomycin as a "cover" for the operation period is helpful in the prevention of complications due to the spread of tuberculous infection after lung resection. It is arguable that at least as much of the improvement in results is due to more careful selection of cases and to advances in operative technique, but we have not felt sufficiently convinced of this to give up the use of the drug. We have given it by intramuscular injection of two doses of $0.5 \mathrm{~g}$. daily, beginning one week before operation and continuing for six weeks. Toxic manifestations with this dosage have been few. More recently the impression has been gained that a longer period of pre-operative streptomycin combined with P.A.S. has resulted in a smoother post-operative course, and where possible a period of three weeks of pre-operative chemotherapy is given, followed by only three weeks' post-operative treatment.

\section{THE OPERATION}

Anaesthesia is induced by intravenous injection of appropriate amounts of "tubarine" and thiopentone. A cuffed Magill's tube is passed into the trachea, and narcosis is maintained by inhalation of a mixture of equal parts of nitrous oxide and oxygen in a semi-closed to-and-fro absorber system. The effect of the nitrous oxide is reinforced periodically by the injection of further increments of thiopentone, and sufficient tubarine is given to abolish or suppress reflex motor responses. Natural respiration is allowed to return gradually at the end of the operation, and 
should be fully restored by the time the skin incision is being sutured. The recovery of consciousness and the cough reflex should also be attained at the end of the operation.

The surgical technique employed differs only in detail from the standard methods of lobectomy and pneumonectomy with dissection and division of the individual structures of the hilum. The most important principle to be observed is that tuberculous infection should not be spread to other parts of the lungs, or to the pleural cavity or the chest wall. Therefore the lung is handled with gentleness, and transection of diseased tissue is avoided. It is of importance to posture the patient so that any secretion extruded from the diseased area cannot reach other parts of the bronchial tree. For this reason, Overholt and others (1946) have recommended the adoption of the prone position on a specially designed table, and Parry Brown (1948) and Sellors and Hickey (1949) have used the " head-down, face-down " position. We have preferred to use the standard lateral position with a considerable head-down tilt which is maintained until the bronchus has been clamped. Any extruded secretions gravitate down the trachea and can be removed by suction.

Pleural adhesions are often dense over cavity areas, but are usually easily freed extrapleurally, with a much reduced risk of opening the cavity. We have freed the lung in the extrapleural plane at all planned pneumonectomies, and find that this facilitates the procedure.

It is not always easy to decide how much lung should be removed, and palpation of the whole lung is necessary when a lobectomy is contemplated. The position of the main lesion is usually easily determined, and the affected segments are often contracted and relatively airless. When there are small lesions separate from the main one (as, for example, is not uncommonly the case in the apical segment of the lower lobe), it may be difficult to judge the degree of activity. They may, of course, be known to have been active in the recent past, or they may have been unsuspected until their discovery at operation. Nodules which are hard and resemble grains of rice to the touch we have usually considered it safe to leave, but we believe that if they are $0.5 \mathrm{~cm}$. or more in diameter, or if they are elastic or soft in consistency, they are foci of active disease and should not as a rule be left behind. Other things being equal, it is better to remove too much rather than too little tissue.

Segmental resection has been approached with considerable caution, but increasing experience has shown that, where a lesion is confined to a segment or segments, resection of that segment can be undertaken with safety. The establishment of this point has been of great importance, for whereas in the early days transection of lung tissue was considered likely to lead to tuberculous infection this in practice has not been found to be so. Palpation of the lung will show which segments are involved, and, if a useful amount of healthy lung tissue can be left, then resection of the involved segments only is now practised. If a portion of lung feels normal to palpation then it is not affected by the tuberculous process.

Where an. area of segmental disease is adjacent to an intersegmental plane, difficulty may be had in stripping the segment and it is wise to remove a small slice of the neighbouring segment. This may lead to an increase in the air leak, but it will seal off within 48 to 72 hours. Small peripheral nodules of doubtful quiescence have been safely removed by a wedge resection. 
This fear of provoking a spread of tuberculous infection led us in the early stages to be too radical in the removal of lung tissue-for example, sacrificing a whole upper lobe for disease of the apical and posterior segments-but recently a more closely definitive operation has been employed.

Every effort is made to clamp the bronchus at an early stage of the operation, but although this is simple in the case of a pneumonectomy it is fraught with greater danger in a lobectomy, when it is usually safer to ligate and divide some of the vessels first.

Removal of a lobe or lung from an unsatisfactory thoracoplasty has not been as difficult as at first was imagined. The approach has been to resect the anterior end of the fifth rib and then to define the space, either intrapleurally or extrapleurally, underneath the reformed bony layers, which is divided up towards the apex as far as required, and the thorax can then be widely opened. The greatest difficulty has been the detachment of a cavity from the paravertebral gutter, particularly if an apicolysis has previously been performed. On occasion the cavity has been entered, but no untoward event followed this mishap.

In empyema cases there is difficulty in separating the pleura from the chest wall and more particularly from the diaphragm, and in a number we have ruptured the cavity during the procedure. Again the results have been benign, and this we believe to be chiefly due to pre-operative treatment of the pleural cavity by aspiration and the instillation of P.A.S. and streptomycin every other day, often for many weeks until it appears sterile. The stripping of the empyema sac from a healthy lobe, which can be left behind, is relatively simple.

\section{Post-operative Treatment}

In general, the convalescence of patients after lung resection is smooth, and there are, only a few days after operation, a sense and appearance of well-being which are in contrast to the state seen after a thoracoplasty.

The intercostal water-seal drainage catheter inserted at the operation is removed after 48 hours, and aspiration of the chest is performed afterwards as may be necessary (usually three or four times after a pneumonectomy, and fewer after a lobectomy). We try to keep the level of fluid below the bronchial stump after a pneumonectomy, at least during the first two weeks, but if clotting takes place this may be impossible to achieve.

Pneumoperitoneum fills are started again a week after operation, and as much air is given as the patient will comfortably tolerate in order to obtain a rapid rise of diaphragm.

Post-operative physiotherapy is important, and is mainly directed to breathing exercises and postural drainage. The rather energetic regime usual after a thoracoplasty is not necessary after a resection operation.

In the absence of any complication, the patient is allowed out of bed two weeks after operation. Each week his daily time out of bed is increased by one hour, and if his condition remains satisfactory exercise is begun when he is up for three hours. After 12 weeks the patient should be up for about 12 hours, and he should be ready for discharge.

When the home conditions are satisfactory a considerable number of patients have been sent home after six weeks under the care of the chest physician and the 
private doctor, reporting to the surgical unit in three months, six months, and one year. This procedure has been found to work satisfactorily.

The Prevention of Hyper-expansion of the Remaining Lung Tissue

As has already been indicated, it is important to bear in mind the condition of the remaining lung tissue when a lung resection has been performed for tuberculosis. According to our present concepts, a tuberculous focus in lung tissue that is under any kind of strain is not in a good condition for healing; collapse therapy is designed to relieve any such strain. There is thus a danger of lighting up activity in a tuberculous focus left behind in lung tissue which undergoes over-expansion after a resection operation. It is possible that this is only a theoretical argument, but in any case it is the better from the point of view of function if the remaining lung tissue can be prevented from expanding beyond its normal size.

After a pneumonectomy the vacant intrathoracic space can be diminished either by a thoracoplasty or by phrenic paralysis and pneumoperitoneum (Figs. 1, 2, and

a

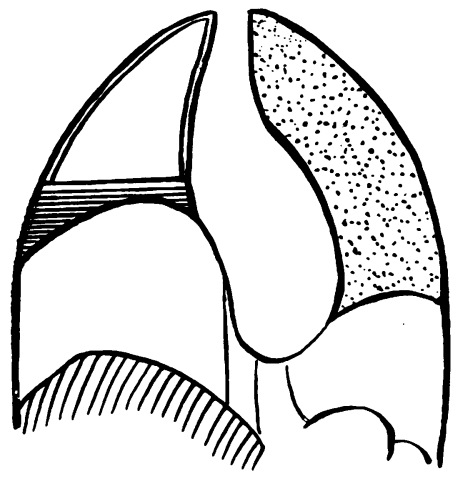

Prenicectomy and Preumoperitoneum after Rught Pheumonectomy.

c

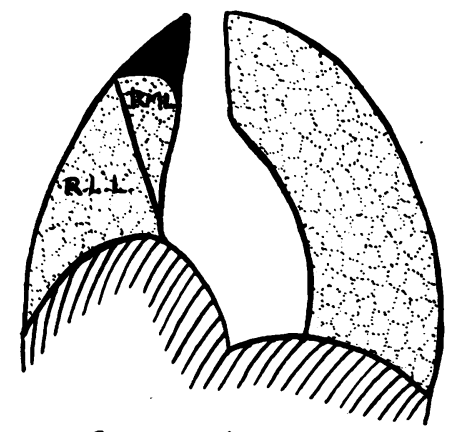

Phrenicectomy

ofter Right Upper Lobectorny. b

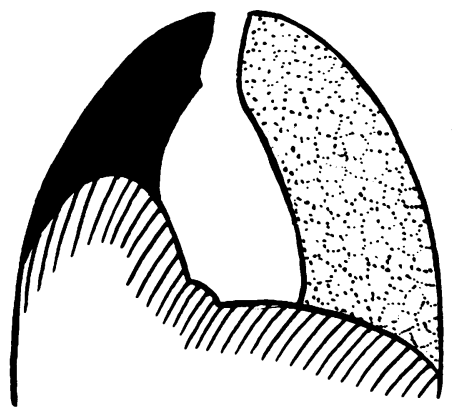

After Abandonment of Pneumoperitoncuin.

d

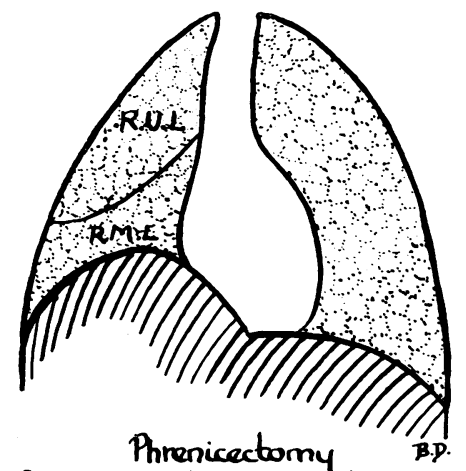

ofter Right Lower Lobectomy.

FIG. 1.-Methods of reducing size of hemithorax to prevent hyper-expansion of remaining lung tissue.

$\mathrm{C}$ 
Fig. 2(a).-Unsatisfactory right artificial pneumothorax with de-aerated upper lobe and "honeycomb" cavities.

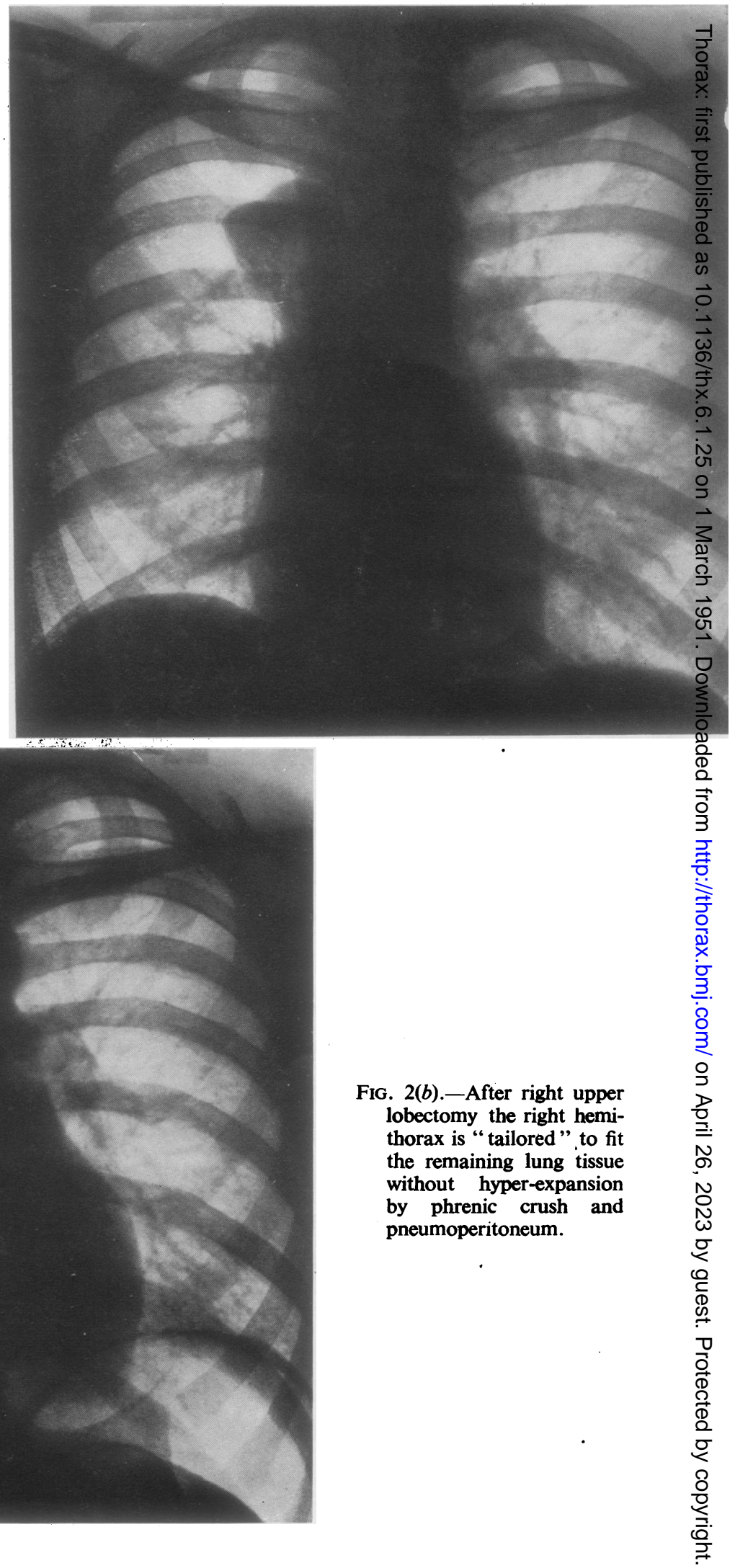


FIG. 3(a).-Fibro-cavernous disease of right upper lobe.

FIG. 3(b).-Right upper lobectomy performed. Capacity of right hemithorax diminished by phrenic crush supplementing the pneumoperitoneum.

FIG. 3(c).-After absorption of the pneumoperitoneum the diaphragm remains high.

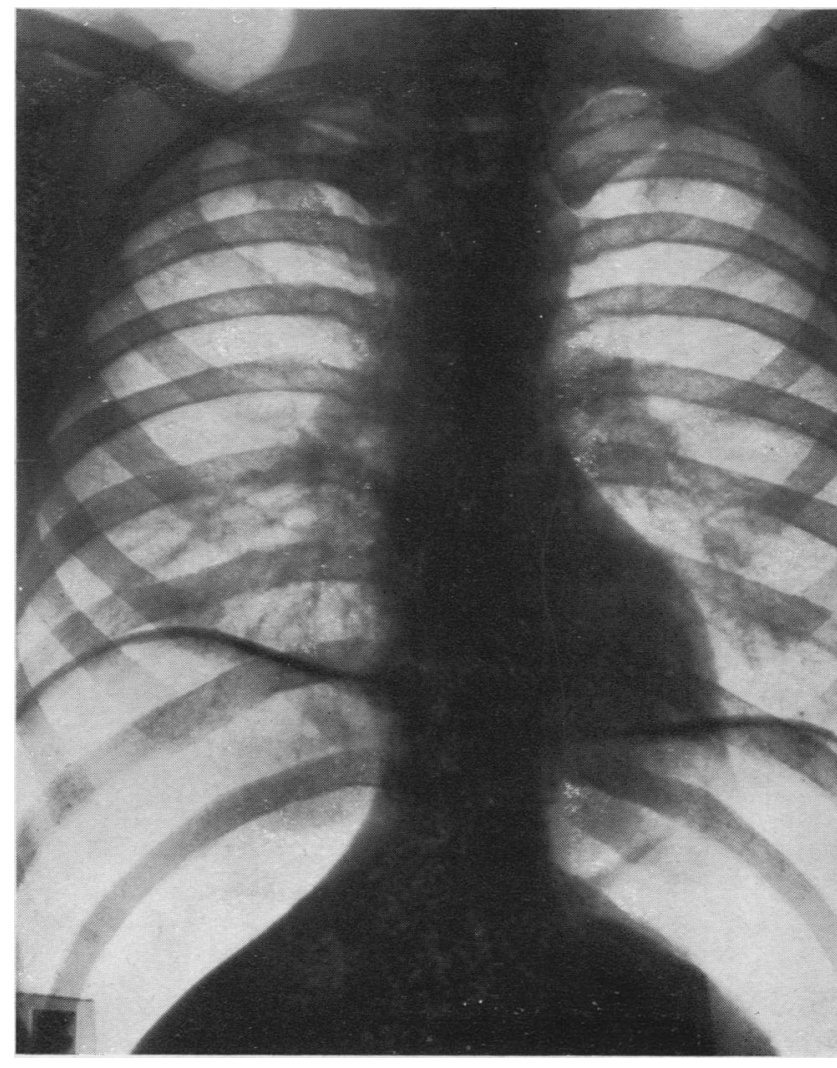

FIG. 3(a)

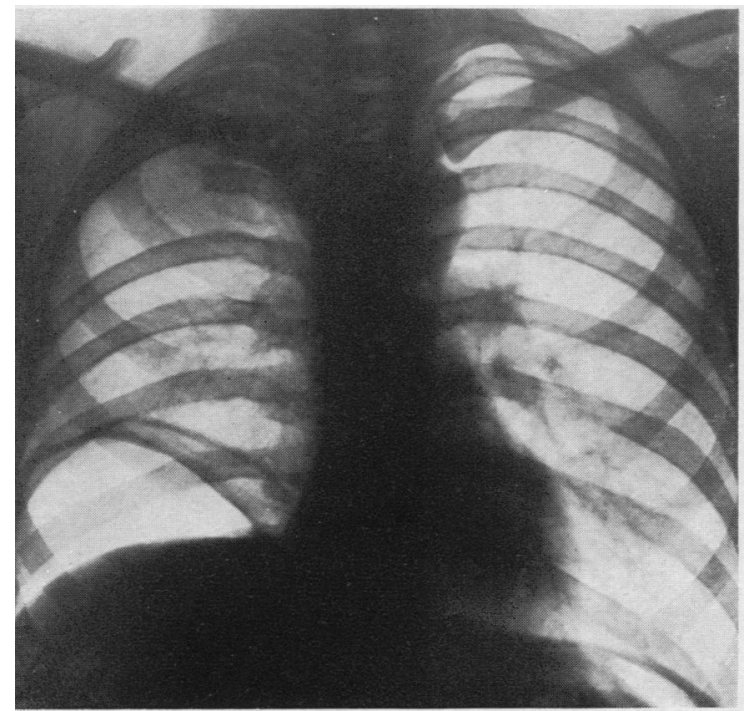

FIG. (3b)

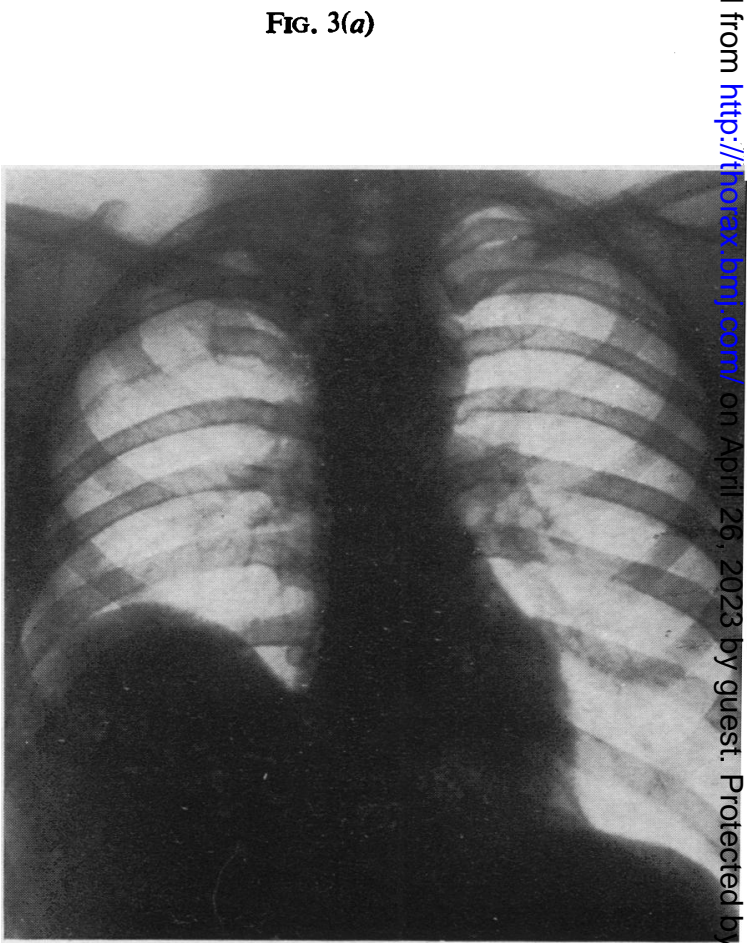

Fig. 3(c) 
3). We have preferred to undertake the latter procedure as a routine, the phrenic nerve being divided in the chest at the time of operation, and have found that a very effective diminution in the size of the space can be obtained. The pneumoperitoneum is maintained for six weeks post-operatively, and by this time the diaphragm appears to become almost fixed in its new elevated position. The hemithoracic cavity eventually fills with fluid which becomes fibrinous, organizes, and contracts to a certain extent; the mediastinal displacement has usually only been slight.

When an upper lobe has been removed the remaining lobes are mobilized by the division of all adhesions and of the pulmonary ligament up to the inferior pulmonary vein. This permits the more even expansion of the lobes as they fill the upper part of the chest.

A difference of opinion occurs as to when the phrenic nerve should be crushed after lobectomy. One of us (F.R.E.) prefers to crush it at the time of operation and another (B. J. B.) prefers to wait until the seventh day post-operatively. No significant resultant differences have been observed between the two procedures.

The persistence of a fibrinous mass after a small collection of fluid at the apex of the chest is not looked on by us with disfavour, as it limits the degree of expansion of the remaining lobe or lobes.

When post-resectional thor-

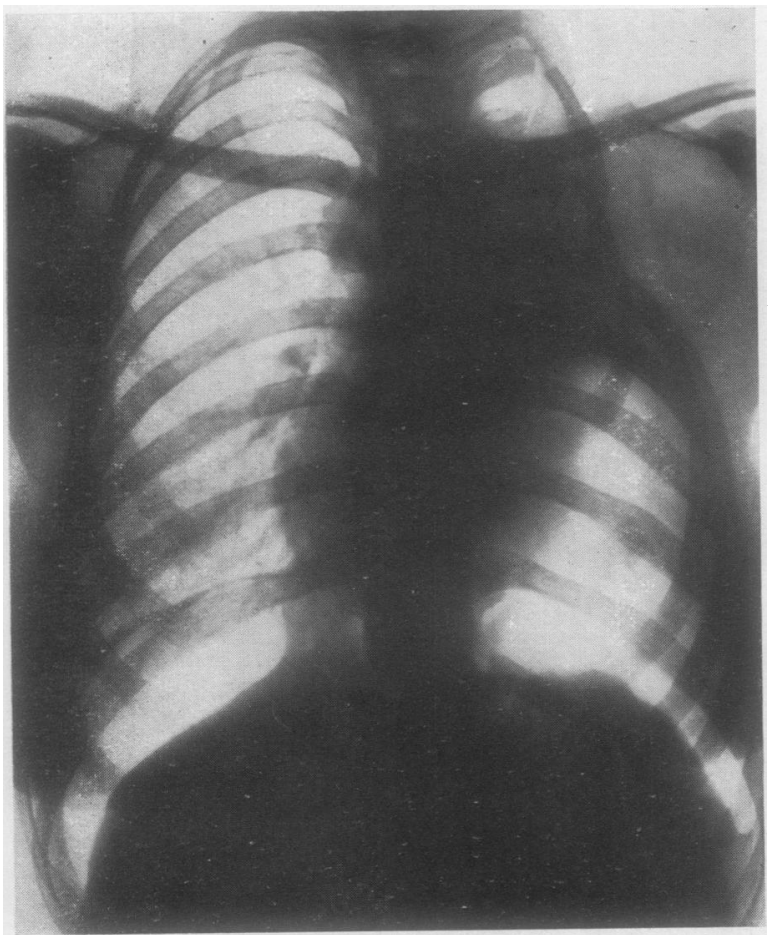

FIG. 4.- - Left pneumonectomy supplemented by a corrective thoracoplasty at the same time as a phrenicectomy and a pneumoperitoneum. The left hemithorax is now much reduced in size (three weeks post-operatively). 
the posterior $5 \mathrm{~cm}$. of the fourth, third, and second ribs and sometimes the sixth rib if this has not already been divided. The first rib is left intact. Minimal external visible deformity of the thorax is produced, with a marked reduction in the size of the intrathoracic space.

Further experience will be needed before the usefulness of this procedure can be fully evaluated, but we believe it has many advantages, especially if combined with the other methods which we have described.

\section{Complications}

To date we have undertaken over 250 resections for pulmonary tuberculosis at the Aintree and Broadgreen centres.

Two hundred of these can be considered to have passed the immediate postoperative stage (three months) and can be reviewed from the point of view of post-operative complications.

The type of lesion in these cases is shown in Table I.

\section{TABLE I}

\begin{tabular}{|c|c|c|c|c|c|c|c|}
\hline Caseous nodule or " & rculoma" & & & $\cdots$ & & & $16(8 \%)$ \\
\hline Tuberculous bronchie & & .. & .. & .. & . & . & $8(4 \%)$ \\
\hline Fibro-caseous disease & . & . & . & .. & $\ldots$ & . & $155(77.5 \%)$ \\
\hline Tuberculous empyem & .. & $\cdots$ & .. & .. & . & $\ldots$ & $8(4 \%)$ \\
\hline Failed thoracoplasty & .. & .. & .. & .. & .. & .. & $13(6.5 \%)$ \\
\hline
\end{tabular}

Table II shows the type of operation undertaken and the major complications encountered.

TABLE II

\begin{tabular}{|c|c|c|c|c|c|}
\hline . & & & & 1 st 100 & 2nd 100 \\
\hline 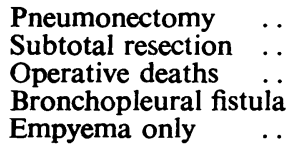 & $\begin{array}{l}\cdots \\
\cdots \\
\cdots \\
\cdots\end{array}$ & $\begin{array}{l}\ldots \\
\cdots \\
\cdots \\
\cdots\end{array}$ & $\begin{array}{l}\cdots \\
\cdots \\
\cdots \\
\cdots \\
\cdots\end{array}$ & $\begin{array}{r}57 \\
43 \\
2 \\
6 \\
4\end{array}$ & $\begin{array}{r}37 \\
63 \\
3 \\
1 \\
1\end{array}$ \\
\hline
\end{tabular}

Five patients died within three months of operation, giving a mortality rate of $2.5 \%$.

Details of these cases are as follows.

Case 1.-Right upper lobectomy after a failed thoracoplasty. Man aged 43. Patient's condition was poor before operation, but could not be improved. Death occurred nine days after operation from pulmonary oedema.

Case 2.-Right pneumonectomy. Woman aged 28. Death occurred seven days after operation from persistent tachycardia. Pre-operatively tachycardia had been noted, but she had been judged fit to stand the operation. Necropsy revealed no definite cardiac lesion and the pulmonary condition was satisfactory.

Case 3.-Left pneumonectomy. Woman aged 35. Death occurred three weeks after operation from massive pulmonary embolism. Necropsy showed condition of remaining right lung to be entirely satisfactory. 
Case 4.-Right pneumonectomy. Woman aged 33. She was a severe diabetic and developed a bronchopleural fistula and an empyema. An old lesion at the apex of the left lower lobe which had been judged stable rapidly reactivated, producing an acute spread of disease throughout the left lower lobe. Death occurred 12 weeks after operation.

Case 5.-Left pneumonectomy. Woman aged 30. Died 36 hours after operation of acute pulmonary oedema of the right lung, which started some six hours after operation. No cause for this could be found at necropsy.

\section{EMPYEMa AND BRONCHOPLEURAL Fistula}

Empyema and bronchopleural fistula are the most distressing complications, although only one patient has died.

The type of empyema is shown in Table III.

TABLE III

\begin{tabular}{|c|c|c|c|c|c|}
\hline & & \multicolumn{2}{|c|}{1 st 100} & \multicolumn{2}{|c|}{ 2nd 100} \\
\hline & & $\begin{array}{l}\text { Pneumon- } \\
\text { ectomy }\end{array}$ & $\begin{array}{l}\text { Subtotal } \\
\text { Resection }\end{array}$ & $\begin{array}{l}\text { Pneumon- } \\
\text { ectomy }\end{array}$ & $\begin{array}{l}\text { Subtotal } \\
\text { Resection }\end{array}$ \\
\hline $\begin{array}{l}\text { Tuberculous empyema } \\
\text { pleural fistula } \ldots \\
\text { Coccal empyema with } \\
\text { fistula } \\
\text { Simple coccal empyema }\end{array}$ & $\begin{array}{l}\text { with broncho- } \\
\text { bronchopleural } \\
\text { broncr } \\
\begin{array}{ccc} \\
\ldots & \ldots & \ldots\end{array}\end{array}$ & $\begin{array}{l}1 \\
4 \\
3\end{array}$ & $\begin{array}{l}1 \\
0 \\
1\end{array}$ & $\begin{array}{l}0 \\
1 \\
0\end{array}$ & $\begin{array}{l}0 \\
0 \\
1\end{array}$ \\
\hline
\end{tabular}

The improvement in the results in the second 100 cases is notable and in the main is due to improved technique. There is still the possibility of late empyema or fistula developing, particularly in the second series, and these figures cannot be taken as final.

Only two of the cases showed evidence of a tuberculous infection. The divided end of the bronchus has been examined histologically in all and evidence of a tuberculous infection has been found in less than $10 \%$ of the specimens.

The occurrence of an empyema, whether with a bronchopleural fistula or not, necessitates further surgery, first drainage and then usually a thoracoplasty. In only one case, a lobectomy, was drainage without thoracoplasty sufficient to effect a cure. The final closure of the empyema sinus may be a long procedure and tedious to both patient and surgeon.

\section{Post-operative Staining of the Sputum}

This is a feature not infrequently seen after lobectomies or segmental resections and appears to be due to blood from the pleural space entering the bronchial tree from the "stripped" surface of the neighbouring lobe or segment. It may persist for 10 to 14 days, but appears to subside without incident. A haematoma in the raw area of lung tissue may be seen in the radiographs. Absorption may take some weeks.

\section{Clotted Haemothorax}

Clotted haemothorax usually follows a reactionary haemorrhage from an area where the pleura has been stripped from the chest wall and is unpleasant rather than 
serious. Aspiration is difficult, and absorption takes place only slowly. The endresult shows some pleural thickening and the function of the remaining lung tissue on the affected side may be reduced. We have not been unduly concerned, however, by the long-term results in these cases, and, although we have been tempted to open the chest and empty out the clot on one or two occasions, conservative treatment has been satisfactory. After a pneumonectomy, provided the clot remains sterile, the supportive action on the mediastinum has been to the patient's advantage.

\section{Lung CollaPse}

Collapse of the lower lobe after an upper lobectomy is infrequent, but if it occurs it is not serious and the lung re-expands rapidly with postural drainage. The problem is different from collapse associated with a thoracoplasty, where the lower lobe is constantly fed with infected sputum from the retained upper lobe with consequent danger of a new area of tuberculous infection developing.

\section{Follow-up of the First One Hundred Cases}

The first 100 cases had been operated on for six months or more on June 1 , 1950, and were reviewed after that date. The length of time since operation was

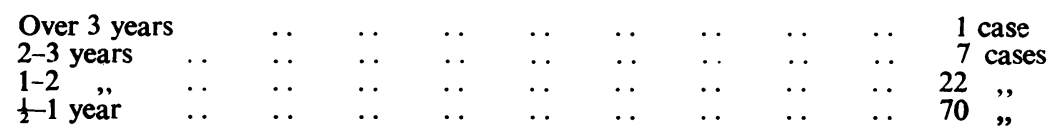

Two patients had died, Cases 1 and 2 of the operative deaths described previously, leaving 98 alive.

Of the ten patients who had a pleural infection, six still had a draining sinus following thoracoplasty, usually only a track, but it was impossible to state when this would be closed.

Pyrexia of uncertain origin was present in two cases. In one, a pre-existent fever was not affected by excision of the left upper lobe containing several nodular lesions, and the site of the persistently active lesions is not known. In the other case a continual low temperature followed an upper lobectomy, and the cause of this cannot be determined. The sputum in both these cases is negative to all tests for tubercle bacilli.

The progress of cases in which there has been evidence of activity of their disease, either clinical, radiological, or bacteriological, at any time after operation is of paramount importance in considering the effectiveness of treatment of pulmonary tuberculosis by any method, and is of particular interest after lung resection.

The details are summarized in Table IV.

In all these patients, except one, where radiological evidence of extension of disease is present, the extension appears to be due to reactivation of an old focus which had been judged to be stable. In no case, as far as we can ascertain, has an extension of disease become established in a new area, suggesting a "spill-over" at the time of operation.

In the group with transient activity, there was no clinical or radiological sign of activity apart from a single positive sputum in three cases; in one other a contralateral apical infiltration cleared completely and has remained satisfactory for two and a half years. 
TABLE IV

Cases with Evidence of Activity of Disease at Any Time after Operation

1. Transient Activity

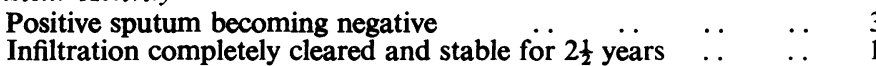

$\begin{array}{r}3 \\ 1 \\ \hline 4\end{array}$

2. Disease Active at Time of Review

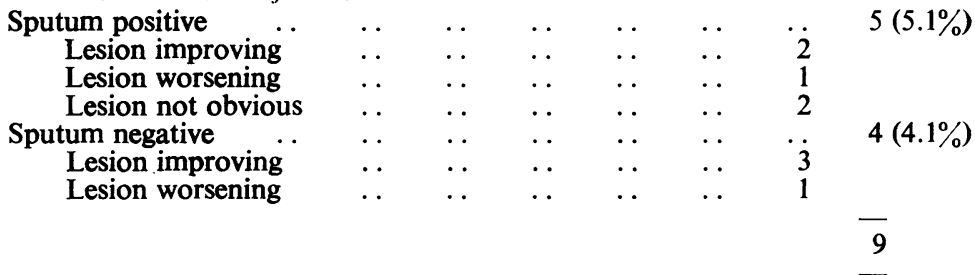

There are nine cases with evidence of activity of disease at the time of review, five with a positive and four with a negative sputum. Of the patients with a positive sputum, there are two with progressive disease, one of whom has a gross exacerbation three years after a lower lobectomy for tuberculous bronchiectasis; the other has been uncooperative from the beginning and has now refused further treatment. Two patients have a positive sputum without any clinical, radiological, or bronchoscopic evidence of disease.

Of the patients with evidence of activity but without a positive sputum, three have slowly enlarging nodular lesions which were present before operation. They are being closely observed at the present time.

Two cases, one of which still has a positive sputum, are regressing under treatment.

Thus of 98 survivors of the operation there are five with a positive sputum at the present time (conversion rate of $94.9 \%$ ), and another four have some activity of their disease without a positive sputum. In other words, $90.8 \%$ of the survivors of the operation have no evidence of activity of their disease at the time of review.

Sputum examination has been made in all cases by culture and in most by guinea-pig inoculation.

The number of patients who had completed at least one year after operation on June 1, 1950, is 30 , and details are shown in Table V.

TABLE $V$

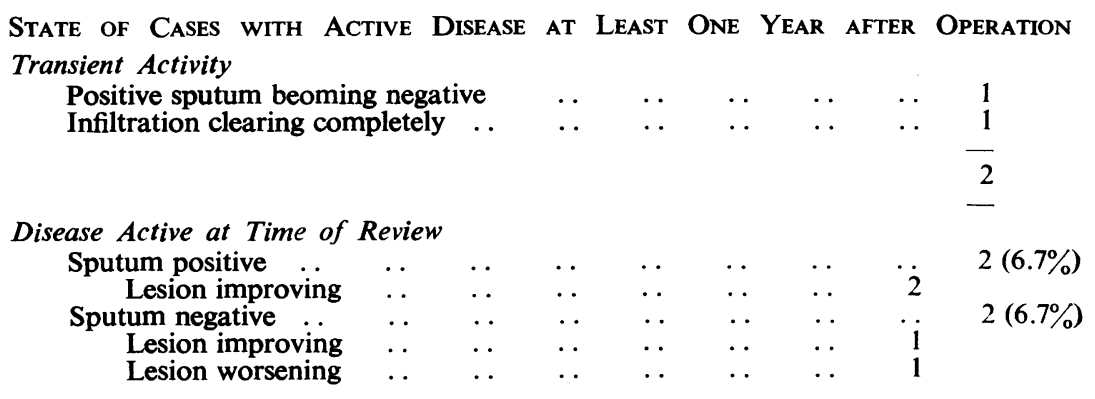


Out of the 100 cases reviewed the outlook must be considered doubtful in the nine cases with evidently active disease, but in only two of them is the disease progressive, uncontrolled, and serious.

What the future will bring forth, as these cases are followed up, we do not know. They are being observed, and the results of the further follow-up study will be published in due course. We are of the opinion at the present state of investigation that the preliminary results of the use of lung resection as an elective method of treatment of pulmonary tuberculosis, when major surgery is indicated, are encouraging and merit an extended trial. In view of the present ultimate mortality of the disease the indications for resection at an earlier stage of the disease may be extended.

\section{SUMmARY}

In addition to the generally accepted indications for resection of tuberculous lung tissue, an investigation has been undertaken to find out if this principle can be generally applied to the treatment of localized active tuberculous disease, failing the development so far of a specific antibiotic.

The operative mortality rate is $2.5 \%$ in 200 cases.

A follow-up of 100 cases that have been operated on for six months or longer is given, and the disease appears to have been arrested in $90.8 \%$.

Our most grateful thanks are due to Dr. Peter O. Leggat, Medical Registrar to the Thoracic Surgical Unit at Broadgreen Hospital, who followed up the Broadgreen cases; to Dr. V. C. Cornwall, Deputy Physician Superintendent at Aintree Hospital, for his continued follow-up of the Aintree Hospital cases; to Messrs. C. Stathatos, J. K. B. Waddington, and H. V. Wingfield, who have operated on some of the cases, and to the very many chest physicians who have given us encouragement in our work.

The results we have achieved are due to the skill of the nursing staff of the two centres, and we should like to record our very sincere appreciation and admiration for their devoted care of these patients.

Bradbury, F. C. S. (1946). The Prevention and Treatment of Tuberculosis in the Administrative County of Lancaster. Preston.

Brown, A. I. P. (1948). Thorax, 3, 161.

Churchill, E. D., and Klopstock, R. (1943). Ann. Surg., 117, 641.

Cox, G. L. (1936). The Prevention and Treatment of Tuberculosis in the Administrative County of Lancaster. Preston.

Dolley, F. S., and Jones, J. C. (1940). J. thorac. Surg., 10, 102.

Gale, J. W., Dickie, H. A., and Curreri, A. R. (1949). Amer. Rev. Tuberc., 59, 10.

Hurford, J. V. (1941). Lancet, 1, 693.

Iverson, R. K., and Skinner, H. L. (1950). J. thorac. Surg., 19, 491.

Livingstone, R. (1949). Tubercle, Lond., 30, 79.

Macewen, W. (1906). Brit. med. J., 2, 1.

Overholt, R. H., Langer, L., Szypulski, J. T., and Wilson, N. J. (1946). J. thorac. Surg., $15,384$.

Wilson, N. J., Szypulski, J. T., and Langer, L. (1947). Amer. Rev. Tuberc., 55, 198.

Sarot, I. A. (1949). Thorax, 4, 173.

Sellors, T. H. (1947). Ibid., 2, 216.

and Hickey, M. P. (1949). Ibid., 4, 82.

Stocks, P., and Lewis-Faning, E. (1944). Brit. med. J., 1, 581.

Sweet, R. H. (1946). J. thorac. Surg., 15, 373.

(1950). Ibid., 19, 298.

Thompson, B. C. (1942). Tubercle, Lond., 23, 139.

(1943). Brit. med. J., 2, 721.

[Appendix, page 42 
A P P E N D I X

Condition of First 100 Operated Cases with Minimum Follow-up of One Year

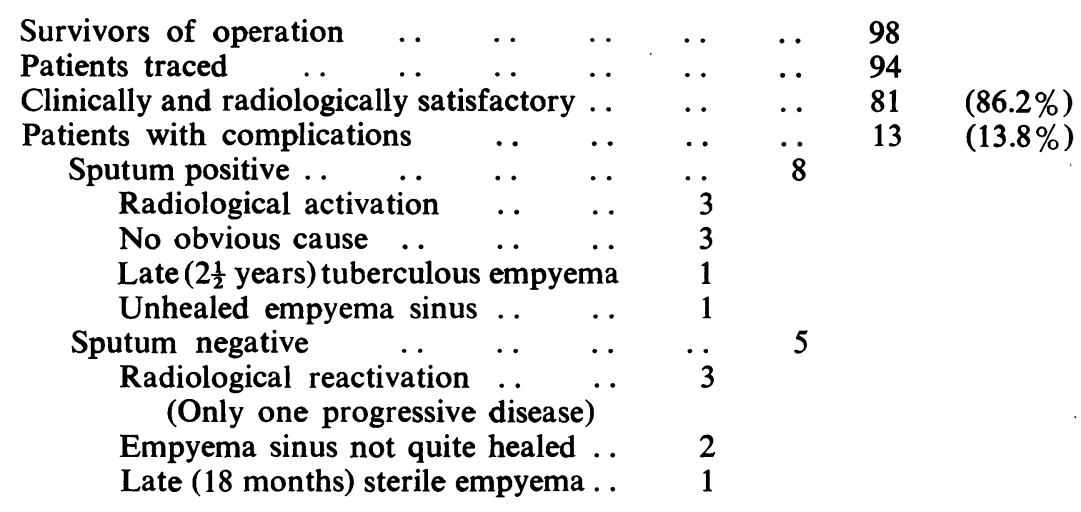

The sputum conversion rate is thus $87.2 \%$ in cases followed for a minimum period of one year after operation, but there is some evidence of activity of disease in 11 cases $(11.7 \%)$. 